\title{
Malformaciones arteriovenosas intracraneales y radiocirugía con LINAC: artículo de revisión
}

\author{
S. Moreno-Jiménez; M.A. Celis-López; J.M. Lárraga-Gutiérrez*; L. Herrera-Gómez; J.J. Suárez-Campos**; A. \\ García-Garduño* y M.Hernández-Bojórquez* \\ Neurocirugía, *Física Médica y **Radio-oncología. Unidad de Radioneurocirugía. Instituto Nacional de Neurología y Neurocirugía MVS. \\ México.
}

Resumen

De las malformaciones vasculares, las malformaciones arteriovenosas (MAV) intracraneales son las que tienen una mayor repercusión desde el punto de vista clínico. Se pueden manifestar con hemorragia, epilepsia, cefalea, o pueden aparecer en forma incidental durante el abordaje diagnóstico de pacientes con traumatismos o cefalea crónica. Existen tres tipos de tratamientos disponibles: microcirugía, embolización y radiocirugía. El papel actual de la terapia endovascular es como adyuvante como tratamiento previo a microcirugía o a radiocirugía para disminuir el tamaño del nido. La meta final de cualquier tratamiento es la obliteración completa del nido sin causar un nuevo déficit neurológico. El índice general de obliteración por radiocirugía con LINAC es alrededor de $80 \%$ y es dependiente de la dosis, del volumen y del tiempo de seguimiento. La dosis promedio publicada en la literatura va de 15 a 25 Gy y la curva de isodosis para conformar la MAV con LINAC es por lo general la del $80 \%$. Puede haber fallo en el tratamiento definido como la necesidad de volver a tratar al paciente después de tres años de seguimiento por falta de obliteración en alrededor del $26 \%$ de los pacientes.

Faltan pruebas principalmente a partir de ensayos clínicos, para seguir definiendo el papel de cada una de las modalidades en el tratamiento integral de las MAV.

PALABRAS CLAVE: Malformaciones arteriovenosas. Radiocirugía. Terapia conformada. LINAC

Intracranial arteriovenous malformations and radiosurgery with LINAC: review article

Summary

Among all vascular malformations, the intracranial arteriovenous malformations (AVM's) have the most

Recibido; 19-08-05. Aceptado: 10-11-05 powerful impact from the clinical point of view. The manifestations include hemorrhage, seizures, headacheh, but sometimes they are incidentally found during the diagnostic approach of patients with head trauma or chronic headache. There are three different types of treatments: microsurgery, endovascular treatment and radiosurgery. The actual role of the endovascular treatment is as an adjuvant therapy before microsurgery or radiosurgery just to diminish the nidus size. The goal of all treatments is complete nidus obliteration without causing a new neurological deficit. The overall obliteration index with LINAC based radiosurgery is about $80 \%$ and the result is dose, volume and time dependent. The mean dose reported in the literature fluctuates between 15 and $25 \mathrm{~Gy}$, and the isodose coverage curve for the AVM with LINAC is generally the one of the $80 \%$. There can be a treatment failure defined as the necessity to retreat the patient after three years from the first radiosurgical treatment in about $26 \%$ of the patients. There is a lack of evidence, principally from randomized trials, to point out the role of each of the modalities in the treatment of the AVM

KEY WORDS: Arteriovenous malformations. Radiosurgery. Conformality. LINAC

\section{Introducción}

Las malformaciones vasculares han sido clasificadas en diferentes formas, pero las más reconocidas son: malformaciones arteriovenosas, angiomas venosos, angiomas cavernosos y telangiectasias ${ }^{7}$. Las malformaciones arteriovenosas (MAV) intracraneales son anomalías congénitas que se desarrollan entre la cuarta y octava semanas de vida intrauterina del embrión. Consisten en la persistencia de una conexión entre una arteria y una vena sin la interposición de una red capilar. Las MAV se pueden manifestar clínicamente de varias formas. La más frecuente es como un accidente hemorrágico intracerebral; sin embargo, también pueden manifestarse con epilepsia, déficit neurológico focal por fenómeno de robo o cefalea. En algunos casos se 
trata de un diagnóstico incidental al estudiar a un paciente por síntomas que no se relacionan con la malformación. La historia natural de las MAV no es conocida por completo ya que no existen grandes series de casos no tratados. El riesgo hemorrágico de las MAV no rotas se encuentra alrededor del 3\% por año ${ }^{4,14}$. Las estimaciones de la prevalencia de las MAV que han sido publicadas en la literatura médica son en general infundadas. Debido a la rareza de la enfermedad y a que existen pacientes asintomáticos, establecer una prevalencia verdadera no es posible. Debido a la variación en el índice de detección de MAV asintomáticas, la estimación más confiable de la ocurrencia de la enfermedad es el índice de detección de lesiones sintomáticas. Esta prevalencia es alrededor de 0.94 por 100,000 personas-año ${ }^{2}$. El tratamiento actual de las malformaciones arteriovenosas cuenta con tres herramientas básicas: resección microquirúrgica, embolización y radiocirugía. El uso adecuado de cada una de ellas, ya sea como modalidad única o en combinación, es necesario para completar un tratamiento con éxito.

La radiocirugía estereotáctica (RE) es hoy en día una atractiva alternativa de tratamiento de los pacientes con MAV. Se han publicado series de pacientes tratados con rayos gamma (Gamma Knife), con partículas pesadas (Ciclotrones) y con rayos $\mathrm{x}$ (aceleradores lineales). Estos últimos han ido creciendo en cantidad alrededor de todo el mundo por lo cual se tiene cada día más experiencia, sin embargo, las publicaciones en la literatura son escasas. En el presente trabajo se da un panorama de las MAV y de las características especiales de la RE con LINAC, además de analizar las diferentes series de pacientes con MAV tratados con esta modalidad de RE.

\section{Radiocirugía}

Existen diferentes aparatos para la realización de radiocirugía como son el ciclotrón, el Gamma Knife y el acelerador lineal (LINAC). La mayoría de las series publicadas de MAV tratadas con este método usan las dos primeras modalidades, sin embargo, cada vez hay más experiencia con los LINAC.

La definición de radiocirugía aún no ha llegado a un consenso general. Pollock y Lunsford definieron la radiocirugía estereotáctica como la administración de una sola dosis de radiación terapéuticamente efectiva a un blanco definido por imagen, y hacen notar la importancia de diferenciarla de la radioterapia estereotáctica fraccionada $^{24}$. Por otro lado, Adler y Colombo, proponen una definición más amplia del término radiocirugía. Mencionan que la definición previamente descrita es innecesariamente restrictiva, y opinan que los principios radiobiológicos de la radioterapia pueden utilizarse para mejorar el tratamiento con radiocirugía. Ellos definen radiocirugía como cualquier procedimiento que incluya la participación de un neurocirujano y en el cual se administre radiación con un método altamente preciso y conformado a un blanco previamente definido con la intención de provocar una lesión ${ }^{1}$. Probablemente la definición que menos confusión provoca es considerar la radiocirugía como dosis única y la radioterapia estereotáctica fraccionada como los demás casos, esto debido a que la mayoría de los artículos de la literatura mundial así lo estiman. Sin embargo no hay que olvidar que existen algunas otras técnicas como es el hipofraccionamiento, opción efectiva y segura para el tratamiento de las MAV de gran tamaño ${ }^{26}$.

\section{Radiobiología}

Para entender la radiobiología de las MAV es necesario comprender primero algunos conceptos generales entre los cuales se encuentra el concepto del modelo lineal cuadrático de muerte celular. El componente lineal, o el componente alfa, es la consecuencia de dos rupturas de un cromosoma, resultado del efecto ionizante de una partícula cargada. La función cuadrática de la dosis, o el componente B (beta), representa la muerte celular que resulta de diferentes partículas cargadas. Para los tejidos de respuesta temprana (tumores malignos), la relación alfa/beta es alta (cerca de $10 \mathrm{~Gy}$ ), mientras que para los tejidos de respuesta tardía (tejido cerebral), la relación alfa/beta es baja (cerca de 2 Gy $)^{17}$.

La radiobiología de la Radiocirugía puede ser caracterizada de acuerdo a si el blanco es de respuesta temprana o tardía y si el blanco contiene tejido normal:

- Categoría I: blanco de respuesta tardía incluido dentro de tejido normal de respuesta tardía, ejemplo, las malformaciones arteriovenosas. El índice alfa/beta es pequeño: existe un efecto radiobiológico similar en ambos.

- Categoría II: blanco de respuesta tardía rodeado de tejido de respuesta tardía, ejemplo, tumores benignos como los meningiomas. Existe un efecto radiobiológico grande dentro del volumen blanco sólo en el tejido anormal.

- Categoría III: contiene un blanco de respuesta temprana incluido en tejido normal de respuesta tardía, ejemplo, astrocitomas de bajo grado en donde el tejido normal y las células tumorales se encuentran dentro del volumen blanco. En este caso, la relación alfa/beta es igual a 10 para el blanco y 2 para el tejido normal. Ambos están expuestos a la misma cantidad de radiación. En esta situación, el efecto radiobiológico de la radiocirugía es experimentado más intensamente en el tejido normal que en el tejido anormal; por lo anterior, la radiocirugía es recomendada en casos seleccionados. - Categoría IV: contiene tejido de respuesta temprana rodeado por tejido de respuesta tardía, ejemplo, el 
glioblastoma multiforme y las metástasis cerebrales. Las células en esta categoría experimentan un gran efecto dentro del tejido anormal, blanco, y un efecto menor en el tejido normal que lo rodea ${ }^{17,18}$.

Para las dos primeras categorías, el índice de curación/ complicaciones es independiente del fraccionamiento (dosis única vs dosis múltiples). El fraccionamiento de la dosis permite que se lleve a cabo una reoxigenación del tejido tumoral haciéndolo más radiosensible. Radiobiológicamente en radiocirugía, gracias a la colimación y conformación que se logra, la dosis liberada alrededor del blanco es, por lo general clínicamente poco significativa, debido a la rápida caída de dosis en la periferia del tumor. En suma, la curva de isodosis mínima al volumen blanco, generalmente de 50\% con Gamma Knife y alrededor del 80-90\% con LINAC, conforma de manera muy estrecha la forma tridimensional del blanco. La experiencia clínica sugiere que cuanto más grande sea un blanco, más pequeña debe ser la dosis, para disminuir el riesgo de complicaciones ${ }^{8}$.

La radiobiología de las malformaciones arteriovenosas ha sido ampliamente estudiada. Debido a que los modelos radiobiológicos describen una relación directa entre el daño tardío del tejido normal y la dosis por tratamiento administrada a los tejidos; varios estudios han apoyado la utilización de la radioterapia estereotáctica hipofraccionada para el tratamiento de malformaciones grandes , $^{90,15,19,31}$.

\section{Patología}

Los cambios vasculares inducidos por la radiación dentro del sistema nervioso central incluyen fibrosis perivascular, necrosis fibrinoide de la pared vascular, degeneración hialina, edema, telangiectasias, trombosis y hemorragia ${ }^{29}$. En las MAV los cambios más frecuentes de los vasos de mediano y pequeño calibre ocurren en la íntima. Estos se manifiestan por edema y vacuolización de las células endoteliales. Posteriormente hay proliferación de células endoteliales y depósito de lípidos lo que lleva finalmente a la trombosis. Schneider y colaboradores estudiaron los cambios histológicos en 10 especimenes de pacientes con MAV tratados con radiocirugía. Las MAV mostraron cambios tempranos, incluyendo edema de las células endoteliales y denudación del endotelio o separación de la línea endotelial de la capa vascular subyacente. Algo importante para la eventual oclusión del vaso es la aparición de células fusiformes organizadas laxamente dentro de la íntima de la pared vascular y la producción de una matriz extracelular que contiene colágeno tipo IV. La expansión progresiva de la íntima, incluyendo la proliferación de las células de músculo liso y engrosamiento de las capas media y adventicia, causan un estrechamiento concéntrico de la luz del vaso. Después ocurre una degeneración celular progresiva con aumento de la densidad de la pared vascular. La trombosis y la fibrosis no son el mecanismo de la oclusión vascular, más bien lo es la expansión de la pared vascular ${ }^{28}$.

\section{Características de los LINAC}

La construcción de los aceleradores de electrones o aceleradores lineales (LINAC) es el resultado de la búsqueda de otros equipos de irradiación que pudieran superar los defectos de otras fuentes de energía como los aparatos de cobalto. El LINAC produce rayos-X de alta energía producto de la colisión de los electrones acelerados con materiales con números atómicos grandes. Los rayos-X producidos por un LINAC cubren un rango amplio de energías desde unas docenas de kilo-electronvoltios $(\mathrm{keV})$ hasta la energía máxima de los electrones acelerados, la cual varía según la aplicación y el equipo de 4 hasta 23 millones de electronvoltios (MeV), a diferencia de la fuente de rayos gamma, con una energía promedio de $1.25 \mathrm{MeV}$. La nomenclatura que se utiliza para identificar la energía de los rayos-X producidos en un LINAC es la energía nominal, la cual se representa con la energía de los electrones acelerados en millones de voltios (MV). Este amplio rango de energías se traduce en la mayor capacidad de penetración de los rayos-X en el tejido. Para los tratamientos radioquirúrgicos con LINAC la energía estándar que se utiliza es de $6 \mathrm{MV}$. Aunque todavía se debate sobre la superioridad del LINAC sobre la radiocirugía gamma, se acepta de forma general que el LINAC posee las siguientes ventajas: 1) No necesita de una fuente natural de radiación, por lo que la tasa de dosis no depende de la vida media del material radiactivo (como el cobalto del Gamma-Knife). Además de que no se tiene el problema de desechar las fuentes cuando éstas ya no sean eficaces para aplicar los tratamientos, así como el costo de su reemplazo. 2) Los equipos modernos de LINAC acoplados con colimadores micromultiláminas sólo requieren de un isocentro para el tratamiento de lesiones irregulares, por lo que no se necesita la recolocación del paciente, reduciendo los tiempos de tratamiento. 3) Se pueden tratar lesiones de mayor tamaño y con esquemas de fraccionamiento (radioterapia estereotáctica fraccionada), lo que amplía el rango de aplicaciones y su versatilidad. Con el Gamma Knife por lo general se requieren varios isocentros para cubrir una sola lesión, además de que para MAV grandes se ha sugerido utilizar un tratamiento en etapas lo cual es algo limitante en la actualidad.

Sin embargo, es necesario comentar que el Gamma Knife es fabricado por una sola casa comercial lo cual le confiere una precisión mecánica en muchos casos mejor que la del LINAC, el cual es producido por varias compañías al igual que los sistemas de colimación y el software utilizado. Esto puede provocar discretas variaciones en los resultados. El LINAC requiere controles y pruebas mecá- 
Tabla 1

Dosis de tratamiento de las series publicadas tratadas con LINAC

\begin{tabular}{llll}
\hline \multicolumn{1}{c}{ Autor } & Año & Dosis (Gy) & Curva de isodosis (\%) \\
\hline Beth y cols & 1989 & $20-70$ & 80 \\
Colombo y cols & 1994 & $18.7-40$ & $70-90$ \\
Friedman y cols & 1995 & $10-25$ & $70-90$ \\
Friedman y cols & 1996 & 15 & $70-80$ \\
Schlienger y cols & 2000 & 25 & $50-90$ \\
Pedroso y cols & 2004 & $12-18$ & $55-90$ \\
\hline
\end{tabular}

nicas rigurosas de precisión como por ejemplo la prueba de Winston-Lutz.

\section{Dosimetría}

Betri y col utilizaron colimadores circulares con un rango de dosis muy amplio, de 20 a 70 Gy, utilizando dosis de no más de 40 Gy en el $80 \%$ de los pacientes ${ }^{3}$. La dosis utilizada por Friedman tiene una media de 15,05 Gy, a mayor volumen menor es la dosis. La línea de isodosis que utiliza más frecuentemente para conformar la MAV es la del $80 \%$, aunque ocasionalmente utilizaba la de $70 \%$ con lo cual encontró un mayor riesgo de sangrado ${ }^{12}$. En otra publicación utilizó dosis con un rango entre 10 Gy y 25 Gy con una media de 15,60 Gy. La curva de isodosis utilizada fue con mayor frecuencia nuevamente la del $80 \%$, con un rango del $70 \%$ al $90 \%{ }^{11}$. Colombo utilizó colimadores circulares y publicó un rango de dosis que va de 18,70 Gy a 40 Gy con una media de 28,2 Gy. La dosis de tratamiento se conformó con las curvas de isodosis del $70 \%$ al $90 \% 5$. El promedio de dosis en la periferia por el grupo de DeSalles fue de 15 Gy con un rango de 12 a 18 Gy utilizando colimadores micromultiláminas para la conformación ${ }^{21}$. Schlienger y col utilizaron colimadores circulares y de uno a 5 isocentros para la conformación de la lesión, y publicaron dosis en la periferia de 15 a 28 Gy con una mediana de 25 Gy. La conformación se realizó con las curvas del 50 al $90 \%$ con una mediana de $70 \%$. La media de dosis, en la lesión fue de 14 a 36 Gy con una mediana de 29 Gy con una media de isodosis en la lesión entre 53 y $90 \%$ con una mediana de $79 \%$. La dosis mínima en la lesión fue de 3.6 a 23 Gy con una mediana de 16 Gy. La curva de isodosis mínima en la lesión fue de 9 a $65 \%$ con una mediana de $45 \%{ }^{27}$. Las dosis utilizadas en las diferentes series se muestran en la tabla 1.

Es importante mencionar la importancia de intentar exponer de una manera más homogénea las diferentes series de pacientes tratados con radiocirugía. Es necesario explicar el tipo de técnica de colimación: conos o multihojas, número de isocentros, número de arcos, métodos de imagen utilizados para la delineación, la dosis de prescripción, la dosis de cobertura, curva de isodosis, índices de conformidad e índices de homogeneidad. De esta manera será más fácil hacer una comparación entre las series.

\section{Resultados de obliteración}

La meta principal del tratamiento de pacientes con malformaciones arteriovenosas es la obliteración de la misma. Esta es la única manera de asegurar la ausencia de riesgo de resangrado. En general se considera que la presencia de una angiografía negativa es igual a una disminución importante del riesgo de resangrado. Por lo general, una vez tratado el paciente, se le hace un seguimiento con imagen de resonan cia magnética y angiorresonancia cada cierto tiempo que puede variar, por lo general, cada seis meses durante los primeros tres años. Una vez que se observan cambios sugerentes de obliteración de la MAV se le solicita una angiografía con sustracción digital para su comprobación. Betti y colaboradores lograron una obliteración en 27 de 41 pacientes con un seguimiento de al menos 24 meses (65.8\%). El porcentaje de curación de pacientes fue significativamente mayor cuando la malformación estaba incluida en su totalidad en la curva de isodosis del 75\% (96\% de curación), y cuando el diámetro máximo de la lesión era menor a 12 mm $\left(81 \%\right.$ de curación ${ }^{3}$. Friedman y col publicaron 158 pacientes con MAV tratados con LINAC con una media de seguimiento de 33 meses. Lograron obliteración completa en un $69 \%$ a $89 \%$ de los casos, dependiendo del volumen de la malformación. Con lesiones menores de $4 \mathrm{cc}$ la obliteración fue del $81 \%$, con lesiones entre 4 y $10 \mathrm{cc}$ fue del $89 \%$, mientras que para malformaciones mayores de 10 cc fue del 69\%. La curación angiográfica general fue del $80 \%$. La conclusión fue que las malformaciones mayores 
Tabla 2

Seguimiento, obliteración y resangrado de las series publicadas tratadas con LINAC

\begin{tabular}{llccc}
\hline \multicolumn{1}{c}{ Autor } & Año & Seguimiento (meses) & Obliteración (\%) & Resangrado (\%) \\
\hline Beth y cols & 1989 & 24 & 65.8 & 4.9 \\
Colombo y cols & 1994 & 24 & 80 & 4.8 \\
Friedman y cols & 1995 & 33 & $69-89$ & 4 \\
Friedman y cols & 1996 & - & - & $5-7$ \\
Schlienger y cols & 2000 & $48-96$ & 64 & 2.4 \\
Pedroso y cols* & 2004 & $37.4-57.4$ & $28.6-84.6$ & 6.8 \\
\hline
\end{tabular}

*En esta serie la gran variabilidad en el porcentaje de obliteración se debe a que dividieron a los pacientes en grupos de acuerdo a la clasificación de Spetzler-Martin. A mayor grado menor porcentaje de obliteración.

de $10 \mathrm{cc}$ pueden ser tratadas con radiocirugía con LINAC de una manera segura y efectiva ${ }^{11}$. Colombo y col publicaron en una serie de 180 pacientes una obliteración completa de $46 \%$ al primer año y de $80 \%$ en el segundo año a partir del tratamiento ${ }^{5}$. DeSalles y col ajustaron el porcentaje de obliteración de acuerdo al grado de Spetzler-Martin. La obliteración fue decreciendo de $84.6 \%$ (Grados I y II), 58.3\% (Grado III) y 28.6\% (Grado IV). No hubo obliteraciones en las MAV Grado V. Las MAV localizadas en el tallo cerebral se ocluyeron en el $60 \%$ de los casos. El índice de obliteración mostró una tendencia a correlacionarse con el Grado de Spetzler-Martin ${ }^{21}$. La obliteración lograda por Schlienger y colaboradores fue en general del $64 \%$ siendo ésta mayor en los casos con volúmenes menores o iguales a $42 \mathrm{cc}$ y con dosis entre $24.1 \mathrm{y}$ 25.9 Gy a la periferia. Después de un análisis multivariado encontraron que la ausencia de embolización previa y la utilización de un solo isocentro fueron factores independientes de buen pronóstico ${ }^{27}$. Los resultados de obliteración de las diferentes series se muestran en la tabla 2.

\section{Resangrado}

La historia natural de las MAV muestran un riesgo de sangrado de $4 \%$ anual ${ }^{20}$. El resangrado es una de las complicaciones más temidas en estos pacientes. Es conocido que la obliteración de la MAV en pacientes tratados con radiocirugía es un proceso paulatino. Existe un periodo de latencia durante el cual el riesgo de sangrado existe.

Betti y col publicaron resangrado y muerte en dos pacientes a los 18 y 29 meses después del tratamiento ${ }^{3}$. Friedman y col aportaron una serie de 214 MAV tratadas, encontrando que el riesgo de sangrado durante el periodo de latencia es mayor mientras mayor volumen tenga la MAV. En un análisis multivariado no se encontró un aumento significativo en el riesgo de sangrado explicado por otras covariables. El riesgo de sangrado durante el periodo de latencia después de la radiocirugía fue del $5 \%$ al $7 \%$ por lo que no se alejó de manera significativa del riesgo por la historia natural ${ }^{12}$. DeSalles y col tienen tres pacientes $(6.8 \%)$ con resangrado después de uno, 14 y 54 meses a partir del tratamiento21. Schlienger y col tienen un índice de resangrado de $2.4 \%$ con una muerte ${ }^{27}$. Cabe mencionar que hay una publicación de pacientes con MAV con resangrado después de haber sido confirmada su obliteración mediante angiografía con sustracción digital, especialmente en los que se presentaba un reforzamiento con medio de contraste en el estudio de TC o RM de control ${ }^{30}$. Debido a que este estudio tuvo un seguimiento limitado, el significado para predecir hemorragia después de la evidencia angiográfica de obliteración es aun indeterminable. Esto nos obliga a continuar el seguimiento de los pacientes a pesar de una angiografía negativa. (Tabla 2)

\section{Fallo en el tratamiento}

Para evitar un fallo en el tratamiento es indispensable cubrir toda la lesión con la dosis de radiación necesaria para provocar los cambios vasculares responsables de la obliteración de la MAV. Esto se logra utilizando diferentes estudios de imagen para conocer la arquitectura de la MAV lo más real posible. Se utilizan diferentes estudios para aprovechar las ventajas de cada uno de ellos. La TC $\mathrm{y}$ angiotomografía sirven principalmente para tener una referencia con marcas fiduciarias con un pequeño índice de distorsión. La RM y la angiorresonancia se usan por su gran resolución espacial lo que permite visualizar de una manera mejor la MAV, sin embargo, su índice de distorsión es mayor que el de la TC. Finalmente la angiografía con sustracción digital permite conocer mejor la arquitectura de 
la MAV, pero con el inconveniente de que existe una sobreestimación del volumen de la misma debido a que se trata de un estudio bidimensional. Se pueden aprovechar todos estos estudios mediante los programas que existen para fusión de imágenes. La delineación de la MAV se realiza básicamente en la angiotomografía, RM, angiorresonancia $\mathrm{y}$ angiografía con sustracción digital.

La definición de fallo en el tratamiento aún no está clara, pero en general podemos considerarla cuando es necesario darle un retratamiento al paciente. Ellis y col explican que en el $26 \%$ de los pacientes que requirieron nuevo tratamiento, el nido sobrepuesto en el plan de tratamiento inicial mostraba que no estaba cubierto. En un análisis multivariado encontraron que los factores relacionados con el fallo en el tratamiento fueron un mayor tamaño de la MAV, disminución de la dosis de tratamiento, y un mayor grado de Spetzler-Martin ${ }^{6}$. Gallina y colaboradores afirman la necesidad de incluir la totalidad de la MAV en el blanco para evitar un fallo en el tratamiento. Un error en la determinación de la forma y tamaño de la MAV por una definición imprecisa del nido por una angiografía incompleta (que no incluya llenado por la arteria carótida externa) es una de las causas de fallo. Una recomendación que hacen es intentar cubrir la totalidad de la lesión en el blanco aunque se tenga que disminuir la dosis total ${ }^{13}$. Es importante evitar la técnica de irradiación parcial del volumen de la MAV o también llamado tratamiento en etapas para evitar fallos. Debemos mencionar el fenómeno de hiperemia oclusiva provocado por la trombosis de las venas de drenaje que lleva a cambios hemodinámicos locales en el cerebro adyacente a la MAV después de una radiocirugía ${ }^{23}$.

\section{Comparación con microcirugía}

Las malformaciones arteriovenosas han sido tradicionalmente tratadas con microcirugía. La microcirugía tiene la ventaja de ofrecer una protección inmediata contra el sangrado.

Después de una radiocirugía existe un periodo de latencia que puede durar hasta dos o tres años. Porter y col hicieron un estudio tanto clínico como de costo/beneficio en pacientes con MAV pequeñas y clasificadas como operables tratados con microcirugía o con radiocirugía. En este grupo selecto de pacientes obtuvieron un beneficio clínico de la microcirugía sobre la radiocirugía. La razón de esta ventaja es que la primera protege de una forma más rápida y efectiva contra la hemorragia. En relación al costo, demostraron que la microcirugía es más cara que la radiocirugía con una diferencia de 6937 dólares por paciente, sin embargo, son necesarios estudios especiales para análisis de costos en pacientes que sean comparables ${ }^{25}$. Pikus y col compararon a 72 pacientes operados por su grupo con pacientes de otras series tratados con radiocirugía. Basados en este análisis, el tratamiento microquirúrgico de pacientes con MAV grados I a III de Spetzler-Martin fue superior a la radiocirugía estereotáctica ${ }^{22}$. Sin embargo, son necesarios más estudios, principalmente ensayos clínicos para sacar conclusiones con un alto índice de certeza.

\section{Conclusiones}

Existen tres herramientas terapéuticas para las MAV: microcirugía, terapia endovascular y radiocirugía. La meta final de cualquier tratamiento es la obliteración del nido sin causar un nuevo déficit neurológico. Es importante conocer la radiobiología de los tejidos normales del sistema nervioso central así como el comportamiento de los tejidos patológicos de acuerdo a la categoría a la que pertenecen. Las MAV pertenecen a la categoría I de Larson y son de respuesta tardía. En general es preferible llamar radiocirugía al tratamiento de dosis única y radioterapia estereotáctica fraccionada a los demás. El LINAC es un aparato que permite emitir rayos $\mathrm{X}$ de alta energía los cuales van a interactuar con los tejidos provocando, en última instancia, la obliteración de los vasos del nido de la MAV. El índice general de obliteración por radiocirugía con LINAC es alrededor del $80 \%$ y es dependiente de la dosis, el volumen y el tiempo de seguimiento. Existe un periodo de latencia durante el cual el riesgo de sangrado existe. La dosis promedio publicada en la literatura va de 15 a 25 Gy y la curva de isodosis para conformar la MAV es por lo general la del $80 \%$. Puede haber fallos en el tratamiento definidos como la necesidad de tratar de nuevo al paciente después de tres años de seguimiento por falta de obliteración en alrededor del $26 \%$ de los pacientes. El papel actual de la terapia endovascular es como tratamiento previo a microcirugía o a radiocirugía. La microcirugía es la primera opción de tratamiento en la actualidad para MAV grado I y II aunque se requieren protocolos de ensayos clínicos para confirmarlo. La radiocirugía es la primera opción para MAV profundas o MAVde grados bajos pero con alto riesgo quirúrgico. Faltan estudios para seguir definiendo de una mejor manera el algoritmo de tratamiento de las MAV.

\section{Bibliografía}

1. Adler, J.R., Colombo, F., Heilbrun, M.P., Winston, K.: Toward an Expanded View of Radiosurgery. Neurosurgery 2004; 55: 1374-1376.

2. Berman, M.F., Sciacca, R.R., Pile-Spellman, J., et al.: The Epidemiology of Brain Arteriovenous Malformations. Neurosurgery 2000; 47: 389-397.

3. Betel, O.O., Munari, C., Rosler, R.: Stereotactic Radiosurgery with the Linear Accelerator: Treatment of Arteriovenous Malformations. Neurosurgery 1989; 24:,311-321.

4. Brown, R.D., Wiebers, D.O., Forbes, G., et al.: The 
natural history of unruptured intracranial arteriovenous malformations. J Neurosurg 1988; 68: 352-357.

5. Colombo, F., Pozza, F., Chierego, G., Casentini, L., De Luca, G., Francescon, P.: Linear Accelerator Radiosurgery of Cerebral Arteriovenous Malformations: An Update. Neurosurgery 1994; 34: 14-21.

6. Ellis, T.L., Friedman, W.A., Bova, F.J., Kubilis, P.S., Buatti, J.M.: Analysis of treatment, failure after radiosurgery for arteriovenous malformations. J Neurosurg 1998; 89: 104-110.

7. Félix, I.A.: Tumores y Malformaciones de los Vasos Sanguíneos. México; Auroch, 2000; pp. 1-26.

8. Flickinger, J.C., Shell, M.C., Larson, DA.: Estimation of complications for linear accelerator radiosurgery with the integrated logistic formula. Int J Radiat Oncol Biol Phys 1990; 19: 143-148.

9. Flickinger, J.C., Lunsford, L.D., Kondziolka, D., et al.: Radiosurgery and brain tolerance: An analysis of neurodiagnostic imaging changes after gamma knife radiosurgery for arteriovenous malformations. Int J Radiat Oncol Biol Phys 1992; 23: 19-26.

10. Flickinger, J.C., Kondziolka, D., Lunsford, L.D., el al.: A multi-institutional analysis of complication outcomes after arteriovenous malformation radiosurgery. Int J Radiat Oncol Biol Phys 1999; 44: 67-74.

11. Friedman, W.A., Bova, F.J., Mendenhall, W.M.: Linear accelerator radiosurgery for arteriovenous malformations: the relationship of size to outcome. J Neurosurg 1995; 82: 180-189.

12. Friedman, W.A., Blatt, D.L., Bova, F.J., Buatti, J.M., Menderhall, W.M., Kubilis, P.S.: The risk of hemorrhage after radiosurgery for arteriovenous malformations. J Neurosurg 1996; 84: 912-919.

13. Gallina, P., Merienne, L., Meder, J.F., Schlienger, M., Lefkopoulos, D., Merland, J.J.: Failure in Radiosurgery Treatment of Cerebral Arteriovenous Malformations. Neurosurgery 1998; 42: 996-1004.

14. Itoyama, Y., Syouzaburou, U., Kuratzu, J., et al.: Natural course of unoperated intracranial arteriovenous malformations: Study of 50 cases. J Neurosurg 1989; 71: 805-809.

15. Kjellberg, R.N.: Stereotactic Bragg peak proton beam radiosurgery for cerebral arteriovenous malformations. Ann Clin Res 1986; 18: 17-19.

16. Larson, D.A., Flickinger, J.C., Loeffler, J.S.: The Radiobiology of Radiosurgery. Int J Radiat Oncol Biol Phys 1992; 25: 557-561.

17. Larson, D.A., Bova, F., Eisert, D., et al.: Consensus statement on stereotactic radiosurgery quality improvement. Int J Radiat Oncol Biol Phys 1993; 28: 527-530.

18. Larson, D.A., Liden, K., Sarby, B.: Irradiation of small structures through the intact skull. Acta Radiol Ther Phys 2001; 21: 591-599.

19. Marks, L.B.: Conventional fractionated radiation therapy vs radiosurgery for selected benign intracranial lesions (arteriovenous malformations, pituitary adenomas, and acous- tic neuromas). J Neurooncol 1993; 17: 223-230.

20. Ondra, S.L., Troupp, H., George, E.D., et al.: The natural nistory of symptomatic arteriovenous malformations of the brain: a 24-year follow-up assessment. J Neurosurg 1991; 73: 387-391.

21. Pedroso, A., DeSalles, A.A.F., Tajik, K, et al.: Novalis Shaped Radiosurgery of arteriovenous malformations. J Neurosurg 2004; 101: 425-434.

22. Pikus, H.J., Beach, M.L., Harbaugh, R.E.: Microsurgical treatment of arteriovenous malformations: analysis and comparison with stereotactic radiosurgery. J Neurosurg 1998; 88: 641-646.

23. Pollock, B.E.: Occlusive Hyperemia: A Radiosurgical Phenomenon? Neurosurgery 2000; 47: 1178-1184.

24. Pollock, B.E., Lunsford, L.D.: A Call to Define Stereotactic Radiosurgery. Neurosurgery 2004; 55: 1371-1373.

25. Porter, P.J., Shin, A.Y., Detsky, A.S., Lefaive, L., Wallace, M.Ch.: Surgery versus Stereotactic Radiosurgery for Small, Operable Cerebral Arteriovenous Malformations: A Clinical and Cost Comparison. Neurosurgery 1997; 41: 757-766.

26. Saunders, W.M., Winston, K.R., Siddon, R.L., et al.: Radiosurgery for arteriovenous malformations of the brain using a standard linear accelerator; rationale and Technique. Int J Radiat Oncol Biol Phys 1988; 15: 441-447.

27. Schlienger, M., Atlan, D., Lefkopoulos, D., et al.: LINAC radiosurgery for cerebral arteriovenous malformations : results in 169 patients. Int J Radiat Oncol Biol Phys 2000; 46: 1135-1142.

28. Schneider, B.F., Eberhard, DA., Steiner, L.E.: Histopathology of arteriovenous malformations after gamma knife radiosurgery. J Neurosurg 1997; 87: 352-357.

29. Schultheiss, T.E., Stephens, L.C.: Invited review: Permanent radiation myelopathy. Br J Radiol 1992; 65: 737-753.

30. Shin, M., Kawahara, N., Maruyama, K., Tago, M., Ueki, K., Kirino, T.: Risk of hemorrhage from an arteriovenous malformations confirmed to have been obliterated on angiography after stereotactic radiosurgery. J Neurosurg 2005; 102: $842-848$.

31. Veznedaroglu, E., Andrews, D., Benítez, R., et al.: Fractionated Stereotactic Radiotherapy for the Treatment of Large Arteriovenous Malformations with or without Previous Partial Embolization. Neurosurgery 2004; 55: 519-531.

Moreno-Jiménez, S.; Celis-López, M.A.; Lárraga-Gutiérrez, J.M.; Herrera-Gómez, L.; Suárez-Campos, J.J.; GarcíaGarduño, A.; Hernández-Bojórquez, M.: Malformaciones arteriovenosas intracraneales y radiocirugía con LINAC: artículo de revisión. Neurocirugía 2006; 17: 317-324.

Correspondencia postal: Dr. Moreno-Jiménez Sergio. Instituto Nacional de Neurología y Neurocirugía MVS. Unidad de Radioneurocirugía. Insurgentes Sur \#3877. Col. La Fama Tlalpan. C.P. 14269 México D.F. 
Comentario al trabajo: Malformaciones arteriovenosas intracraneales y radiocirugía con LINAC: artículo de revisión de Moreno Jiménez y cols

El trabajo del Dr. Sergio Moreno Jiménez está bien escrito y es bastante explicativo, tanto en cuanto a los aspectos radiobiológicos de las MAV como en cuanto a la descripción de las distintas técnicas radioquirúrgicas aplicables a este tipo de patología vascular cerebral. Además, se celebra muy especialmente que está escrito en castellano, lo cual no es muy común en tanto que el tema que estudia viene escasamente recogido en la literatura de habla hispana. No obstante, y puesto que de un artículo de revisión se trata, se echa de menos, quizás, alguna ilustración de los especimenes histológicos descritos en la literatura, así como aprovechar el trabajo para aportar, aunque brevemente, la propia experiencia personal de los autores contrastada también con la abundante casuística internacional, sobre todo la pionera del hospital Karolinska de Estocolmo, tanto en referencia a los artículos clásicos de Leksell y Steiner, como a la más reciente de Karlsson o Lindqvist. Bien es cierto que el trabajo se cierne especialmente a los aspectos vinculados a la radiocirugía con LINAC, pero incluso así, permítaseme advertir la conveniencia de haber incluido en el repertorio bilbiográfico algunos otros artículos de revisión previos, entre los que citaría, especialmente, el de puesta al día canadiense ${ }^{2}$ que revisa extensamente en forma de tabla las experiencias de muchas series de casos desde 1985 hasta el inicio de la década actual. Igualmente, y en lo que se refiere al algoritmo de tratamiento, sugeriría un repaso de otro buen artículo de revisión que tiene, además, la ventaja de postularse como una propuesta de "guía" para el tratamiento de estas MAV y que ha venido patrocinada, como estudio cooperativo que es, por la International RadioSurgery Association (IRSA) ${ }^{1}$.

\section{Bibliografía}

1. L. Dade Lunsford, M.D., et al. (The Radiosurgery Guidelines Committee). Stereotactic Radiosurgery for Patients with Intracaranial Arteriovenous Malformations (AVM). Radiosurgery Practice Guideline Initiative. Report \#2-03 (September 2003).

2. Stereotactic radiosurgery: an update. Alberta Heritage Foundation for Medical Research and Health Technology Assessment. (2002).

M.A. Pérez-Espejo Murcia 